\title{
Poétique de la lettre dans les Lettres d'un voyageur
}

L'invention d'un style

\section{Brigitte Diaz}

\section{(2) OpenEdition}

\section{Journals}

Édition électronique

URL : http://journals.openedition.org/recherchestravaux/177

DOI : 10.4000/recherchestravaux. 177

ISSN : 1969-6434

Éditeur

UGA Éditions/Université Grenoble Alpes

Édition imprimée

Date de publication : 15 avril 2007

Pagination : 41-54

ISBN : 978-2-84310-107-7

ISSN : 0151-1874

\section{Référence électronique}

Brigitte Diaz, «Poétique de la lettre dans les Lettres d'un voyageur », Recherches \& Travaux [En ligne],

70 | 2007, mis en ligne le 27 novembre 2008, consulté le 07 septembre 2020. URL : http://

journals.openedition.org/recherchestravaux/177 ; DOI : https://doi.org/10.4000/recherchestravaux. 177 
Brigitte DiAZ

Université de Caen

\section{Poétique de la lettre dans les \\ Lettres d'un voyageur \\ L'invention d'un style}

En dépit de la banalité de leur titre, que Sand emprunte à $\mathrm{M}^{\mathrm{me}}$ de Staël${ }^{\mathrm{I}}$, en dépit de leur forme relativement convenue dans la littérature du temps, les Lettres d'un voyageur constituent une ouvre singulière et originale dans l'ensemble sandien. Ces modestes "Fragments de lettres» - selon le titre originel inscrit de la main de Sand sur le manuscrit des deux premières lettres ${ }^{2}-$ se distinguent, de l'avis de l'auteur elle-même, par le projet qui a présidé à leur écriture: "Je viens donc de relire les Lettres d'un voyageur de septembre 1834 et de janvier I835 ", écrit-elle dans Histoire de ma vie, " et j'y retrouve le plan d'un ouvrage que je m'étais promis de continuer toute ma vie. Je regrette beaucoup de ne l'avoir pas fait ». Cet ouvrage aurait consisté, poursuit-elle, " à rendre compte des dispositions successives de [son] esprit d'une façon naïve et arrangée en même temps ", ou encore, comme elle l'explique dans une métaphore suggestive, à "faire le propre roman de [sa] vie " sans en être le personnage réel, mais « le personnage pensant et analysant ${ }^{3}$ ». La lettre, oscillant entre spontanéité et littérarité, entre introspection et exercice de la pensée, était sans doute la forme d'écriture la plus apte à produire cette biofiction paradoxale, destinée non pas à fournir de nouvelles légendes du moi

I. «Mirza ou Lettre d'un voyageur » est une nouvelle de jeunesse de $\mathrm{M}^{\mathrm{me}}$ de Staël, publiée en 1795 dans Recueil de morceaux détachés.

2. Comme le signale Georges Lubin dans l'introduction de son édition des Lettres d'un Voyageur, op. cit., p. 635 .

3. G. Sand, Histoire de ma vie, édition de G. Lubin, op. cit., vol. II, p. 299. 
mais à lui permettre de se penser une identité plus universelle à travers la figure mobile, à tous égards, du voyageur.

A posteriori ce que Sand retient des Lettres d'un voyageur c'est donc avant tout leur intention, leur élan et leur désir, autant que les questions auxquelles elles s'efforçaient de répondre, bref, ce que j'appellerai leur caractère expérimental. C'était d'ailleurs déjà cette dimension qu'elle privilégiait, en écrivant ces lettres, si l'on en croit le mode d'emploi qu'elle en fournit à Charles Didier, destinataire initial de la lettre $\mathrm{X}^{4}$ :

Ne lis jamais mes lettres avec l'intention d'y apprendre la moindre chose certaine sur les objets extérieurs; je vois tout au travers des impressions personnelles. Un voyage n'est pour moi qu'un cours de psychologie et de physiologie dont je suis le sujet, soumis à toutes les épreuves et à toutes les expériences qui me tentent, condamné à subir toute l'adulation et toute la pitié que chacun de nous est forcé de se prodiguer alternativement à soi-même, [...] à l'enthousiasme ou au dégoût de la vie, au caprice du califourchon, à l'influence du sommeil, à la qualité du café dans les auberges, etc., etc. (P. $893 ;$ p. $271^{5}$.)

"Cours ", « épreuves ", « expériences »..., les mots disent bien de quel type d'expérimentation intellectuelle et ontologique le voyage est l'occasion, ainsi que la lettre chargée d'en faire le rapport; le voyage sandien n'étant à tout prendre qu'une métaphore de l'existence: «Un voyage [...] est un abrégé de la vie de l'homme» (p. $900 ;$ p. 278).

Si voyage il y a, c'est d'abord de ces voyages immobiles que l'on mène davantage sur la page de sa lettre que sur le terrain de ses pérégrinations. À côté de la métaphore pédagogique, c'est d'ailleurs une métaphore littéraire, éditoriale même, dont se sert Sand pour évoquer a posteriori le caractère programmatique de cette ouvre de jeunesse, la présentant comme une "préface " à sa vie d'écrivain: «[...] pour qui s'intéresserait aux secrètes opérations du cœur humain, certaines lettres familières, certains actes, insignifiants en apparence, de la vie d'un artiste, seraient la plus explicite préface, la plus claire exposition de son œuvre» (p. 646; p. 38), écrit-elle dans la préface de l'édition de 1843 , comme pour s'excuser auprès de ses lecteurs, " amateurs de romans ", de ne pas leur livrer leur marchandise habituelle.

Bio-fiction expérimentale, donc, par laquelle George Sand se penche pour la première fois sur son être écrivain, les Lettres d'un voyageur nous intéressent par le double éclairage qu'elles portent sur le chemin déjà parcouru d'une part et sur les voies nouvelles qui se cherchent là d'autre part. Certes, quand

4. Son nom disparaît à partir de l'édition Perrotin en I843.

5. Selon la convention que nous adoptons pour ce volume: G. Sand, Lettres d'un voyageur, pages de l'édition de G. Lubin puis pages de l'édition de H. Bonnet. 
Sand écrit les Lettres d'un voyageur elle n'en est plus vraiment à la préface de sa vie ni à celle de son ouvre, mais déjà à la fin du premier acte. Les deux années et demie sur lesquelles court la rédaction des lettres, I834-1836, ponctuent d'une pause, d'une respiration, le rythme emballé des débuts. Parvenue à un moment charnière de son parcours, George Sand, dans ce débat « polyvoque " que les lettres ouvrent entre elle-même et ses alter ego, cherche à la fois de nouvelles postures " auctoriales ", une nouvelle place dans la convivialité intellectuelle et artistique de son temps, mais aussi de nouvelles harmonies poétiques, hors du roman qu'elle a déjà beaucoup pratiqué. En ce sens, les Lettres d'un voyageur sont des essais, à tous les sens du terme, où l'écrivain prend la distance et le temps nécessaires pour évaluer sa propre pratique et se faire, comme elle dit, "le personnage pensant et analysant ${ }^{6}$ » de son histoire intellectuelle et spirituelle.

En quoi donc le scénario épistolaire ici convoqué prédispose-t-il à cette mise à l'épreuve de soi comme écrivain? Comment s'opère dans et par la lettre une expérience subjective et esthétique fondatrice, ou refondatrice, de la poétique sandienne? Et enfin dans quelle mesure le laboratoire scriptural des Lettres d'un voyageur participe-t-il à l'invention d'un style? Telles sont les questions qui guideront ma lecture.

\section{Lettre et mélancolie}

[...] mais dans mes jours de spleen je marche tranquillement au beau milieu du chemin le plus uni, et je ne plaisante pas avec les abîmes.

Lettre I.

Pourquoi donc la forme épistolaire? La première réponse est triviale et tautologique, c'est qu'à l'origine il s'agit de vraies lettres, du moins pour certaines d'entre elles ${ }^{7}$ - lettres à Musset (lettre I), à Jules Néraud (lettre IV) et à François Rollinat (lettre V). Motivée par l'éloignement du destinateur - le Voyageur - ou par celle du destinataire, la lettre est l'occasion d'une causerie sans façon et elle remplit ici le programme qu'on lui a traditionnellement

6. Voir note $\mathrm{n}^{\circ} 3$.

7. Comme le suggère pour la première lettre cette annonce de Sand dans une lettre à Musset: "Je t'ai écrit une longue lettre sur mon voyage dans les Alpes, que j'ai l'intention de publier dans la revue, si cela ne te contrarie pas. » "À Musset, I5 avril I834 ", Correspondance, édition de G. Lubin, Paris, Garnier " Classiques Garnier », I964-I99I, vol. II, p. 56I-562. 
attribué, à savoir de permettre "une conversation entre des absents »: " Puisque tu ne peux pas venir aujourd'hui, je viens m'enfermer avec toi et causer par la voie de la plume et de l'encre avec ton ennui [...]» (p. 766-767; p. I5I), peut-on lire dans la lettre $V$ adressée à Rollinat, ou encore dans celle que le Voyageur adresse au Malgache: "J'arrive au pays, et je ne t'y trouve plus; une lettre de toi, datée de Marseille, m'arrive presque en même temps. Où vas-tu?» (lettre IX; p. 869; p. 249), suit une longue lettre-journal, comme il y en a quelques-unes dans le recueil. Plus généralement, la lettre, qui trouve son prétexte dans l'éloignement des correspondants, va fournir au Voyageur un cadre d'énonciation commode, apte à recevoir des énoncés variés dans leur forme comme dans leurs enjeux. C'est donc évidemment d'abord pour sa souplesse que Sand utilise ici la forme épistolaire. Hors genre ou plutôt « omnigenre ", la lettre, de par sa plasticité, ouvre un large registre de parole et c'est la raison pour laquelle Sand l'a volontiers adoptée et souvent conseillée aux débutants, notamment à Solange, quand celle-ci se piqua d'entrer en littérature:

La forme des lettres est une des plus commodes pour commencer, on n'est pas obligé de penser au public tant qu'on en est à s'essayer ainsi, et c'est une grande fatigue de moins. Après un an ou deux de cet amusement [...] on peut se réveiller avec une forme et une manière qui s'adaptent à toutes les idées qu'on $\mathrm{a}^{8}$.

Au-delà de cette commodité, la lettre semble avoir ici une autre nécessité: si le Voyageur - qui se présente comme poète, barde, ménestrel, ou encore " serf littéraire » (lettre VI; p. 813; p. 194) - l'utilise, c'est aussi qu'elle met en scène en le fictionnalisant son rapport à l'écriture. Pour qui écrire? Pourquoi écrire? Qu'écrire? Qui suis-je, moi qui écris? sont les questions fondatrices du geste épistolaire dont la lettre elle-même se veut la réponse - et l'on sait combien est obsessionnellement présent dans les lettres intimes ou familières ce métadiscours inquiet. On retrouve dans les Lettres d'un voyageur ce discours d'escorte attendu qui vient questionner ou plus souvent justifier l'acte épistolaire: "Puisque vous me demandez une longue lettre et que vous êtes avide des travaux de la pensée, je veux vous parler de Lavater » (p. 827; p. 208), et voilà légitimée la longue dissertation sur Lavater et la physiognomonie (lettre VII à Franz Liszt). Toujours sommé de fonder la nécessité de sa lettre, c'est son propre rapport à l'écriture que l'épistolier interroge.

C'est d'abord sa séparation d'avec le monde, qui est ou qui fut le sien, que le Voyageur figure et analyse dans sa lettre. Par son éloignement il est brutale-

8. G. Sand, « À Solange, I5 septembre I85I », Correspondance, op. cit., vol. X, p. 429. 
ment livré à l'expérience de sa propre étrangeté au monde: "Me reconnaissez-vous, paisibles Pénates? ce pèlerin qui arrive à pied dans la poussière du chemin et dans la brume du soir, ne le prenez-vous point pour un étranger? ” (lettre V; p. 76I; p. I45). Soi-même comme absent, c'est ainsi que le Voyageur sandien se découvre par l'effet de la distance géographique ou temporelle (les lieux quittés sont aussi du passé cristallisé) que la lettre exacerbe: «Me voilà effacé de sa mémoire " (livre VI; p. 779; p. I62), écrit Sand à propos du Malgache, et cette formule qui prend place dans une anecdote anodine est néanmoins emblématique d'une obsession de l'effacement de soi qui hante les Lettres d'un voyageur et questionne l'incertaine présence au monde du poète. La cérémonie des adieux est une scénarisation parmi d'autres de cet effacement qui peut-être redouté mais aussi consenti, voire désiré par le Voyageur, comme c'est le cas dans la lettre VI à Éverard qui s'achève sur un adieu solennel aux proches: "Adieu, ô mes enfants! j'ai été jusqu'ici plus enfant que vous; je m'en vais seul et loin en pèlerinage, pour tâcher de vieillir vite $[\ldots]$ " (p. 8I7 ; p. 197). Ces adieux s'accompagnent même d'un testament - «Si je ne reviens pas, voici mon testament " - qui dit assez combien l'éloignement du voyage, ou celui que suscite l'écriture, est une mort symbolique. Ce fantasme de l'effacement est vécu de façon plus angoissante encore dans le rêve de la lettre II, où le rêveur se voit peu à peu englouti dans une liquidité mortifere: "Aussitôt que je fais un pas sur la grève, je m'enfonce dans une fange nouvelle $[\ldots] »($ p. 680 ; p. 69). Certes, de cette fange terrifiante il ramène au réveil le «souvenir de quelques lambeaux de vers » où s'explicite le lien entre le scénario fantasmatique de la disparition et la création poétique. Mortifere également, l'attraction du poète voyageur envers son propre passé qui l'absorbe et l'efface du présent: "Pourrai-je vivre demain? Je m'étais rejeté dans le passé, et je savourais cette illusion imbécile au point de me croire transporté aux jours qui sont derrière nous " (lettre IV; p. 740; p. I27). Il voyage dans sa mémoire comme dans un cimetière, interpelle ses chers disparus - « [...] viens me chercher! Déroule ce linceul où $j$ 'ai enseveli ton corps brisé par son dernier sommeil [...]" (lettre IX ; p. 880 ; p. 259) -, et il se représente lui-même errant dans ce " monde des morts qui se meut et s'agite sous le monde des vivants", tel un "fantôme qui porte un nom et des habits, un corps indolent et brisé, une figure terne et pâle» (lettre V; p. 778 ; p. I62).

À d'autres étapes de son parcours, l'effacement du monde des vivants peut être aussi vécu comme une libération. C'est le moment de grâce de la " maison déserte » où, " loin des bruits du monde et des vains discours des hommes ", le nomade se sédentarise pour se faire anachorète. Mais cet enchantement n'est qu'une parenthèse dans l'itinéraire du pèlerin: 
Eh quoi! chère maison déserte, tu veux déjà t'écrouler! tu dureras donc si peu de temps? Asile sacré où j'ai médité, seul et dans le silence, une si douce page de ma vie, seuil hospitalier que je veux baiser [...]. (Lettre VII ; p. 848 ; p. 227.)

Ces motifs de l'effacement, thématisés par la fiction du voyage, interrogent métaphoriquement les rapports ambivalents du poète avec ses contemporains. Le Voyageur qui écrit à l'ami lointain comme le poète qui s'adresse au monde se trouvent l'un comme l'autre confrontés à la douleur de la séparation, l'un par son errance, l'autre par sa vocation qui fait de lui « un oiseau de passage dans la vie humaine9 ». Mais c'est aussi sans le détour de la fiction, que le poète dit de quel arrachement d'avec les siens l'écriture est la cause et le produit:

C'est le malheur de ma destinée, c'est la haine d'autrui qui m'ont fait voyageur et artiste. Moi, je voulais vivre de la vie humaine, j'avais un cœur, on me l'a arraché violemment de la poitrine. On ne m'a laissé qu'une tête, une tête pleine de bruit et de douleur, d'affreux souvenirs, d'images de deuil, d'outrages... (Lettre IX; p. $878 ;$ p. 257.)

Où se rejoignent les deux termes de la métaphore: le voyage et l'écriture qui produisent tous deux une même destinée d'errance et d'exclusion.

À la fois responsable et victime de cet ostracisme, le poète vit son exil comme une sorte de fatalité, parfois même comme un châtiment: "...C'est bien fait! pourquoi étant poète, [...] étant destiné à la tristesse et à la liberté, me suis-je lié à la société? Pourquoi ai-je fait alliance avec la famille humaine? " (lettre IX; p. 877; p. 256). Par ce qu'elle suppose d'absence et de deuil celui de l'autre aimé et du vécu commun - la lettre, et surtout la lettre intime, la lettre-confidence, est plus apte qu'une autre à dire ce clivage ontologique et ce malaise existentiel du poète qui fait de lui, comme il est dit dans la lettre XI, " un être incomplet et maladif » (p. 926; p. 303).

Quant à cette incomplétude du poète dont il est beaucoup question dans les Lettres d'un voyageur, elle met forcément à l'épreuve la légitimité de sa parole. L'artiste, "pauvre diseur de métaphores" (lettre VI; p. 78I; p. I64), fait son confiteor épistolaire. La lettre - entre confession et plaidoyer - a vocation à formuler cette inquiétude parce qu' elle est, pourrait-on dire, culturellement et imaginairement, une forme d'écriture qui entretient avec ellemême un rapport d'incertitude et de doute qu'on perçoit bien dans les éternels clichés formulés par les épistoliers sur leur difficulté à se dire ${ }^{\mathrm{T}}$, que le

9. "Je ne suis qu'un oiseau de passage dans la vie humaine; je ne fais pas de nid et je ne couve pas d'amours sur la terre [...]" (lettre VI ; p. 782 ; p. I65).

IO. "Nos lettres sont incapables de donner une expression satisfaisante de nos propres sentiments, même dans nos meilleurs moments nous sommes forcés de recourir à ces expressions 
Voyageur épistolier convoque lui aussi, s'inquiétant tour à tour de la futilité de ses relations (lettre X; p. 88I ; p. 260) ou encore de l'incohérence de ses lettres soumises au régime arythmique de la digression (lettre II; p. 686; p. $75)$.

La lettre apporte en outre sa dimension d'oralité car on y dialogue et le forum épistolaire devient le lieu d'une évaluation de la voix du poète: qu'a-til à dire de plus ou de mieux que les autres? Comment se fait-il entendre parmi toutes ces voix? C'est là la matière du débat de quelques lettres, notamment la lettre IX au Malgache, qui peut se lire comme un véritable essai sur cet " être à la fois disgracié et privilégié qu'on appelle poète » (p. 873; p. 252), et qui complète la lettre VI à Éverard qui traite, elle, des mérites comparés des artistes et des hommes d'action. Pourquoi ajouter sa voix au monde? La question est au cœur de ce débat virtuel et continu que le Voyageur mène avec lui-même. Aucune voix humaine - ni celle du poète, ni celle du politique - ne semblant pouvoir briser le silence fondamental qui entoure l'humain - ce "silence effrayant, réponse éloquente et terrible de l'éternité " que fait entendre "la grande voix de l'univers" ${ }^{\mathrm{II}}$ ".

Menacé par ce silence éternel, la voix du poète l'est aussi par le bruit des hommes. Inaudible ou surnuméraire, elle est appelée à se perdre dans ce " misérable murmure qui plane sur notre âge [et] n'est qu'un écho de paroles vides et de déclamations sonores " (lettre VIII; p. 855; p. 234-235) - on croirait entendre Lorenzaccio, que Musset invente alors, on est en $1834 \ldots$ Sans compter les voix de la nature qui font concurrence au poète et savent mieux que lui susciter la poésie. Dans une sorte d'oxymore acoustique, Sand fait vibrer autour du voyageur un univers sonore où même le silence devient musical. De prodigieuses polyphonies tour à tour délicates, comme à Venise où " la magie des effets acoustiques" sublime " la plus modeste chansonnette ${ }^{\mathrm{I} 2}$ ", ou assourdissantes comme le "vacarme harmonieux » déchaîné par

comme "indescriptible", "indicible", ou encore à un "si triste", ou "si beau" suivi d'une phrase en "que" vite émiettée. " F. Kafka, Lettres à Milena, Gallimard, I956, p. 260.

II. "Tu lutteras en vain contre la grande voix de l'univers; les astres éternels auront toujours raison, et l'homme, quelque grand qu'il soit parmi les hommes sera toujours saisi d'épouvante quand il voudra interroger ce qui est au-dessus de lui. Ô silence effrayant, réponse éloquente et terrible de l'éternité! » (lettre VI; p. 795; p. I77-I78).

I2. On peut citer plus précisément: «L'absence de chevaux et de voitures et la sonorité des canaux font de Venise la ville la plus propre à retentir sans cesse de chansons et d'aubades. [...] Cette magie des effets acoustiques et le besoin d'entendre une harmonie quelconque dans le silence de ces nuits enchantées font écouter avec indulgence, je dirais presque avec reconnaissance la plus modeste chansonnette qui arrive, passe et se perd dans l'éloignement " (lettre II; p. 698 ; p. 86). 
les orgues de l'église Saint-Nicolas de Fribourg (lettre X; p. 9II ; p. 288), alternent avec un silence tantôt religieux, tel celui qui règne à Torcello, tantôt poétique, comme celui de la jeune fille rencontrée dans les Alpes, bien "plus éloquent et plus profond qu'un livre ${ }^{13} »$. Et toutes ces voix, sources d'enchantements subtils pour le poète, semblent s'allier pour narguer son inanité:

Il y a dans la nature je ne sais quelle voix qui me crie de partout, du sein de l'herbe et de celui du feuillage, de l'écho et de l'horizon, du ciel et de la terre, des étoiles et des fleurs, et du soleil et des ténèbres, et de la lune et de l'aurore, et du regard de mes amis: Va t'en, tu n'as plus rien à faire ici? (Lettre IV; p. 751; p. 137.)

"La voix lointaine et plaintive" (lettre V; p. 759; p. I44) du poète, " homme mélancolique ${ }^{\mathrm{I} 4}$ ", s'entend toujours en dissonance avec ces voix enchantées, et cette mélancolie nous ramène à la lettre, toujours secrètement minée par ses regrets, ses absences et ses deuils... Il y a dans les lettres du Voyageur un itinéraire "spleenétique " qui le mène au " dégoût de tout " (lettre IV; p. 738; p. I25), à l'ennui et l'" atonie intellectuelle" (lettre V; p. 767 ; p. I5I) : " Je suis seul à force de désenchantements et d'illusions perdues " (lettre IV; p. 747; p. I33), écrit Sand dans une formule que, peut-être, Balzac lui a volée.

Moins mélodieuse que les voix du monde, moins éloquente que le silence du monde, que reste-t-il de la voix du poète? Presque rien, un murmure, une " plainte entrecoupée, mêlée de fièvre, de sanglots, de rires lugubres et de jurements " (lettre V; p. 756; p. I4I), qui dit une certaine défaite de la voix poétique.

\section{Polyphonies épistolaires}

[...] la douleur n'embellit que ce qui est beau.

Lettre V.

I3. On peut citer: "Pour moi, je respectais religieusement son silence et ne sentis pas même le désir d'en entendre davantage. Cet amour de la patrie, exprimé, par un mot, par un refus de parler, et par deux larmes bien vite essuyées, me sembla plus éloquent et plus profond qu'un livre. Je vis tout un roman, tout un poème dans la tristesse de cette silencieuse étrangère " (lettre I; p. $658 ;$ p. 48).

I4. Pour citer mieux: "Il n'y a que toi ici, homme mélancolique, créature éphémère et craintive, qui saches quelle heure il est; toi seul comprends cette voix lugubre qui part du clocher et qui coupe ta vie par petites portions égales, sans jamais, s'arrêter ou se ralentir. Va, prends ton bâton et voyage; tu pourras revenir et trouver la maison debout. Telle qu'elle est, elle durera plus que toi [...]» (lettre VII ; p. 849 ; p. 228). 
À privilégier cet « amer savoir » que le poète tire de son voyage, on ferait facilement des Lettres d'un voyageur un texte baudelairien avant la lettre. Or le désenchantement n'est pas le terme d'un voyage qui n'est d'ailleurs pas donné comme achevé. On sait combien Sand a tenu à la disposition de ses lettres dans le volume ${ }^{15}$; la dernière, qui ouvre une polémique avec Nisard et la critique, n'annonce aucune abdication, aucun silence du poète, bien au contraire. Il y a donc une autre cadence du voyage sandien, une autre façon de marcher au bord de l'abîme, en hasardant "les pas les plus gracieux sur les bords de l'Achéron" (lettre I; p. 656; p. 46), comme le Voyageur qui chemine sur la route de Trente, surplombant la gorge de la Brenta. Sur les pérégrinations désenchantées de son personnage, George Sand porte un double regard et chante volontiers la palinodie - la lettre et son inhérente facétieuseté (lettre VI; p. 809; p. 190) le lui permet. L'errance se vit alors davantage comme vagabondage et la plainte devient hymne à la joie:

[...] n'étant bon à rien qu'à causer avec l'écho, à regarder lever la lune et à composer des chants mélodiques ou moqueurs pour les étudiants poètes et les écoliers amoureux, j'ai pris, comme je te le disais hier, l'habitude de faire de ma vie une véritable école buissonnière où tout consiste à poursuivre les papillons le long des haies [...]. (Lettre VI ; p. 784 ; p. I67.)

"Causer avec l'écho ", c'est peut-être là la vraie formule de la poétique sandienne, que le Voyageur découvre chemin faisant, et qui fait des paroles du poète tout autre chose qu'un "écho de paroles vides ". "Causer avec l'écho ", c'est un des premiers scénarios de la création chez George Sand, quand, enfant solitaire sur son balcon madrilène, elle essayait de charmer cette autre "voix qui est dans l'air ${ }^{16}$ ".

L'écho, c'est celui que sa parole cherche et trouve en retour chez l'autre; c'est la réponse, le dialogue, le partage, mais aussi la musicalité, l'harmonie que la voix du poète s'essaye à fonder dans le concert du monde: «[...] chanter et $[\ldots]$ se faire entendre " (Lettre VI; p. 807; p. 189), c'est le vou et la fonction du poète selon Sand. Et ce dialogue des vivants, la lettre a toutes les harmoniques pour le saisir. Il est dans la nature de l'écriture épistolaire de

I5. Voir la lettre du 23 janvier I837 à Buloz: G. Sand, Correspondance, op. cit., vol. III, p. 662.

I6. "Bien heureusement pour moi, elle ne m'expliqua pas ce que c'était que l'écho. Elle n'avait peut-être jamais songé à s'en rendre compte; elle me dit que c'était une voix qui était dans l'air, et l'inconnu garda pour moi sa poésie. Pendant plusieurs autres jours, je pus continuer à jeter mes paroles au vent. Cette voix de l'air ne m'étonnait plus, mais me charmait encore; j'étais satisfaite de pouvoir lui donner un nom, et de lui crier: "Écho, es-tu là? M'entends-tu? Bonjour, écho!" " G. Sand, Histoire de ma vie, édition de G. lubin, op. cit., vol. I, p. 574 . 
fondre la voix de l'un et de l'autre dans un dialogisme fécond, qui se manifeste ici dans toutes les figures de l'échange, du partage, depuis la conversation jusqu'au chant choral, en passant par le débat.

Ce dialogisme est inscrit au programme de la lettre, il est sa raison d'être: "Écrivons-nous tous les jours, je t'en prie; je sens que l'amitié seule peut me sauver " (à Néraud; p. 737; p. I24); il est son horizon, sa récompense: "Tes lettres, il est temps de te l'avouer, font sur moi un effet magique " (à Éverard; p. 813; p. 194). Il est aussi son régime énonciatif ordinaire. L’apostrophe (" Frère me comprends-tu? c'est à ton âme que je parle »; lettre VII; p. 845; p. 224), l'interpellation ("Pourquoi saluons-nous les ruines, les plages inconnues, les neiges immaculées? »; lettre VII; p. 82I-822; p. 203), l'invocation (" Levez-vous, hommes choisis, hommes divins, qui avez inventé la vertu! »; lettre VI; p. 789; p. 172) constituent l'énonciation favorite de l'épistolier qui la module dans des accords volontiers solennels et mystiques. Ses interlocuteurs - qui ne sont pas n'importe qui mais les grands noms de la scène intellectuelle et artistique du temps - Sand ne fait pas que les convoquer, elle rapporte aussi leurs propos, au style direct ou indirect, surtout quand ils s'opposent aux siens, comme ceux d'Éverard, dans la lettre VI, qui conspue au nom de l'engagement politique le " pauvre diseur de métaphores » qu'est le poète, ou encore ceux de Rollinat qui désespère des hommes et des poètes. De méditative, la lettre se fait argumentative pour nourrir un débat sur le poète, sa fonction, son pouvoir ou son impuissance. De ces "entretiens graves ", comme dit Sand, ce n'est pas tant l'argumentaire qu'on retiendra, même si, comme l'écrit Marie d'Agoult à propos de la lettre IX (au Malgache), sa " définition du poète restera ${ }^{17}$ ". Ces réflexions en effet dessinent les figures attendues, en ces temps de désenchantement, du poète prophète qui " élève la voix et dit aux hommes des vérités qui les irritent ", du poète martyr et messie, dont Sand avant Baudelaire chante la paradoxale " bénédiction »:

Le ciel, qui lui a fait une vue si perçante, lui a donné pour la plainte et la bénédiction, pour la prière et pour la menace, une voix abondante et sonore qui trahit imprudemment toutes ses angoisses. (Lettre IX; p. 873; p. 253.)

On peut être plus sensible en revanche aux modulations de la voix du poète, à ses inflexions mélodiques, à ses inventions rythmiques, à cette « spirale har-

17. «Ces lettres intimes ont cela de bon que non seulement elles ajoutent un diamant à votre couronne d'auteur, mais encore qu'elles attachent à votre personne et font aimer en vous la femme. Votre définition du poète restera. » Lettre de Marie d'Agoult à George Sand du Io juin I836, G. Sand et M. d'Agoult, Correspondance, édition de Ch.-F. Dupêchez, Paris, Éditions Bartillat, 1995, p. 59. 
monieuse " (lettre V; p. 759; p. I44) qu'elle trace d'une lettre à l'autre, pour reprendre une métaphore du voyageur, dans la lettre $V$, qui évoque en ces termes la voix plaintive du vent traduisant le désarroi humain.

Il y a dans les Lettres d'un voyageur un autre dialogisme, plus orchestral que discursif, plus polyphonique qu'argumentatif, et qui donne à ces lettres leur timbre musical. Les Lettres d'un voyageur sont habitées par la musique, et pas seulement parce que Sand s'y adresse à des musiciens. La musique y apparaît comme un absolu esthétique: "la musique peut tout exprimer ", c'est le "plus beau de tous les arts» (lettre XI; p. 923; p. 300). Dans la musique, l'esthétique rejoint exemplairement l'éthique: "La musique s'enseigne, se révèle, se répand, se communique. L'harmonie des sons n'exige-t-elle pas celle des volontés et des sentiments? [...] Oui, la musique, c'est la prière, c'est la foi, c'est l'amitié, c'est l'association par excellence ", écrit Sand dans sa lettre à Liszt. Le musicien, qui " vit d'accord, de sympathie, et d'union avec ses élèves »(p. 8I8; p. 199), échappe à la malédiction du poète et suscite autour de lui une communauté sensible et harmonieuse. Ce sont ces harmonies, à la fois acoustiques et humaines, que le pèlerin sandien - barde ou ménestrel - cherche dans ses errances. Son parcours est d'ailleurs essentiellement ponctué des pauses musicales qui modulent des notes d'intensité affectives et esthétiques: Venise, Torcello, Fribourg, la Vallée Noire... Le voyageur sandien est un palimpseste de voix: la voix de Beppa qui s'élève dans la nuit vénitienne comme "l'appel d'une sirène amoureuse " (lettre II; p. 687; p. 76); "le murmure de la Brenta" (lettre II; p. 660; p. 50); le "chant sublime et bizarre, doux comme une prière d'enfant " (lettre II; p. 662; p. 52) de l'ami disparu; le "vacarme harmonieux " de l'orgue de Mooser, dans lequel le Voyageur retrouve «les scène rustiques » de sa vie (lettre X; p. 9II ; p. 288).

La musique dans les Lettres d'un voyageur fait bien plus que nourrir une thématique ou célébrer le culte de la fraternité des arts et dans l'art; elle engage une poétique, dont le rêve de la lettre II pourrait définir allégoriquement le programme. Dans une lagune imaginaire, le poète se voit entouré d'amis inconnus qui " chantent des airs délicieux "; ils lui présentent une " harpe d'une forme étrange » dont il sait pourtant tirer " des sons divins » que tous écoutent " avec attendrissement ». Au réveil ne restent plus dans la mémoire du rêveur que "quelques lambeaux de vers ", à partir desquels cependant, il pourrait écrire, dit-il, " le poème le plus fantastique que le siècle ait encore produit» (p. 680; p. 69).

Ainsi rêvé, le poème est une virtualité musicale, une promesse de bonheur, à vivre dans une communion sensuelle et mystique. Le poète, l'écrivain, 
est pour Sand celui qui s'essaye à recomposer cette harmonie rêvée même s'il désespère de la réinventer jamais. "Je comprends cette langue divine mais ne puis la parler » (lettre VII; p. 845; p. 224-225), écrit Sand dans sa lettre à Liszt, parce cette langue divine qu' elle veut saisir, musicale et immatérielle, redoute avant tout la pesanteur des mots, des formes, des rhétoriques, bref, la littérature. La conversion esthétique de ce que Sand appelle la "vie primitive " risque de n'être jamais qu' un " arrangement de mauvais goût ${ }^{18}$ ", comme elle l'écrit dans l'avant-propos de François le Champi.

De cette poétique quelque peu déceptive, la lettre est un agent précieux. Écriture de l'élan, de la genèse, des commencements, elle capture sans les figer encore en littérature des bribes d'émotions, des éclats de voix et de vie, un peu à la manière de ces Vénitiens, "dilettanti passionnés » mais " ignorants de la musique écrite ", qui "vont recueillant dans leur mémoire les bribes d'harmonie qu'ils peuvent saisir à la porte de palais » et les « cousent à d'autre portions éparses qu'ils possèdent par ailleurs » (lettre II; p. 697; p. 85). La lettre tient de ce " pot-pourri » musical - pour reprendre la métaphore vénitienne où, dans la friction ou la fusion acoustiques de chants et de voix pluriels s'élabore un style.

C'est en cela que les Lettres d'un voyageur sont un champ d'expérimentation. Elles contiennent déjà et exacerbent les éléments essentiels d'un style en devenir, de ce style dont, beaucoup plus tard, Sand fera en quelque sorte la poétique, dans d'autres lettres, notamment celles qu'elle adressera à Flaubert.

Écriture ambulatoire, nomade, la lettre invente et s'invente chemin faisant, comme le voyage qu'elle rapporte, mais aussi comme le roman, tel que Sand le conçoit - un genre libre, ouvert à tous les possibles: "Il en est du voyage comme du roman: ce qui passe est ce qui commande ${ }^{19}$ ", écrit-elle à Flaubert, guère sensible, on l'imagine, à ce nomadisme-là. La lettre assure la liaison métonymique et métaphorique entre voyage et écriture, et plus encore avec l'écriture du roman. Le roman est lui aussi un chemin qui, écrit Sand, " a la permission de faire voyager le lecteur » en lui ouvrant « la faculté de bien

18. Voir l'avant-propos de François le Champi (I847) : «La nature est une œuvre d'art, mais Dieu est le seul artiste qui existe, et l'homme n'est qu'un arrangeur de mauvais goût. " Préfaces de George Sand, édition de Anna Szabó, Debrecen (Hongrie), université de Debrecen Studia Romanica, I997, t. I, p. I42.

19. G. Sand, "À Flaubert, I4 juin I867 ", Correspondance, op. cit., vol. XX, p. 432. 
voir et de jouer de ce qu'il a vu ou de ce qu'il verra ${ }^{20}$ ». Et « qu'y a-t-il de plus beau qu'un chemin? ", demandait le narrateur de Consuelo ${ }^{21}$.

Écriture adressée, la lettre interpelle, apostrophe, appelle l'autre avec fermeté ou tendresse. Sur ce point de sa poétique qui ne variera pas, Sand n'est pas non plus en accord avec Flaubert. Â son dogme de l'adresse restreinte, elle opposera un œcuménisme flamboyant :

On écrit pour tout le monde, pour tout ce qui a besoin d'être initié [...]. Qu'estce que c'est que l'art sans les cœurs et les esprits où on le verse? Un soleil qui ne projetterait pas de rayons et ne donnerait la vie à rien ${ }^{22}$.

C'est déjà le credo professé dans les Lettres d'un voyageur.

Répugnant à la monodie, la lettre accueille les voix de l'autre et réinscrit la parole du poète dans une vaste orchestration. Il y a une polyphonie sandienne, moins réaliste peut-être que celle que Balzac dirige dans La Comédie humaine, mais aussi vraie, et qu'on entend plus qu'ailleurs dans les Lettres d'un voyageur. De cette polyphonie, Sand, à la fin de sa vie, dans une lettre à Flaubert, donne sa formule poétique:

Le vent joue de ma vieille harpe comme il lui plaît d'en jouer. Il a ses hauts et ses bas, ses grosses notes et ses défaillances, au fond ça m'est égal pourvu que l'émotion vienne, mais je ne peux rien trouver en moi, c'est l'autre qui chante à son gré, mal ou bien, et quand j'essaie de penser à ça, je m'en effraie et me dis que je ne suis rien, rien du tout ${ }^{23}$.

Où l'on retrouve la « harpe d'une forme étrange " que le rêveur vénitien avait reçu des mains de ses amis inconnus, pour qu'il en tire " le poème le plus fantastique que le siècle ait encore produit » (lettre II ; p. 680-68I ; p. 69-70).

20. G. Sand, «À Lucien Anatole Prévost-Paradol, I7 octobre I860 », ibid., vol. XVI, p. I52.

2I. G. Sand, Consuelo, La Comtesse de Rudolstadt, Paris, Éditions de la Sphère, 1979, p. 267.

22. G. Sand, "À Flaubert, $\mathrm{I}^{\mathrm{er}}$ octobre $\mathrm{I} 866$ », Correspondance, op. cit., vol. XX, p. I37.

23. G. Sand, "À Flaubert, 29 novembre I 866 ", ibid., vol. XX, p. 207. 